\title{
Frequency Dependent Electrorheological Properties: Origin and Bounds
}

\author{
Hongru Ma,*, Weijia Wen, Wing Yim Tam and Ping Sheng \\ Department of Physics, The Hong Kong University of Science and Technology, Clear Water Bay, Kowloon, Hong Kong
}

(Received 1 March 1996)

\begin{abstract}
We present a unified framework for the first-principles calculation of the frequency dependent shear modulus, static yield stress, and structures of dielectric electrorheological systems. It is shown that a strong (applied field) frequency dependence of the static yield stress, in good quantitative agreement with those measured experimentally, can arise from Debye relaxational effects that are typical of poor insulators. Physical upper bounds on the yield stress and the shear modulus, as well as frequencyinduced structural soft modes, are predicted. [S0031-9007(96)01165-9]
\end{abstract}

PACS numbers: $61.90 .+\mathrm{d}, 41.20 . \mathrm{Cv}, 62.20 .-\mathrm{x}$

Electrorheological (ER) fluids are a class of materials whose rheological characteristics are controllable through the application of an electric field. In this work, we consider a particular type of ER fluids, the dielectric electrorheological (DER) systems, defined as colloidal dispersions of dielectric particles in which the electrical response of both the solid and the liquid components is governed by linear electrostatics. Besides being a topic of general theoretical interest in itself, the DER model has been widely invoked to explain the various aspects of the ER phenomenon, such as the mechanism of chain formation [1], the solid structure under an electric field [2], and the widely observed quadratic field dependence of the yield stress [3]. In spite of these successes, however, serious gaps still exist. Among them are the lack of quantitative understanding for the observed (applied electric field) frequency and conductivity dependencies of the yield stress, and the question concerning the upper bounds of ER shear modulus and yield stress. In the absence of a first-principles account for those issues, the gaps in our understanding are the source of much speculation about the basic mechanism of the ER effect and its potential limitation(s).

In this Letter, we present a general framework for firstprinciples DER model calculations that is based on the formulation of the problem as one of effective dielectric constant optimization. That is, since the operating frequencies of ER fluids are generally $<10^{4} \mathrm{~Hz}$ and the typical particle size and interparticle separation are $<10^{-2} \mathrm{~cm}$, most ER systems are in the "long-wavelength limit," or the electrostatic limit, by a comfortable margin [4]. Provided the components of the system are governed by linear response, the DER model applies, and the electrostatic free energy density is given by $-\bar{\varepsilon}_{z z} E^{2} / 8 \pi$, where $\bar{\varepsilon}_{z z}$ is the component of the effective dielectric tensor along the field $(z)$ direction [5], and $E=\Delta \phi / \ell$ is the applied electric field, where $\Delta \phi$ is the voltage difference and $\ell$ is the length of the sample. The condition of minimum free energy thus directly translates into the maximization of $\bar{\varepsilon}_{z z}$ as a function of particle configurations, plus the consideration of configurational entropy for temperature effects. Below we focus only on the case where the electrostatic energy dominates over the temperature effects. Results of our calculations show that the DER model not only accounts quantitatively for the measured nonlinear (effective) electrical response of ER fluids as well as the measured frequency dependence of the yield stress, but also predicts an interesting frequency-induced structural soft mode that has yet to be observed. By considering the mathematical origin of the frequency dependencies, upper bounds for the yield stress and shear modulus are obtained.

Consider a two-component DER model system consisting of spherical solid particles of radius $R$ and complex dielectric constant $\varepsilon_{1}$ dispersed in a fluid characterized by $\varepsilon_{2}$. We assume the dielectric constants to have the form characteristic of Debye relaxation, i.e., $\varepsilon-\varepsilon_{\infty}=$ $\varepsilon_{\Delta} /(1-i \omega \tau)$, which is commonly observed in poor insulators at low frequencies. Here $\varepsilon_{\infty}$ is the high frequency dielectric constant, $\varepsilon_{\Delta}=\varepsilon(\omega=0), \tau$ denotes the relaxation time, and $\omega$ is the angular frequency. The electrostatic problem to be solved is given by

$$
\boldsymbol{\nabla} \cdot\left[1-\frac{1}{s} \eta(\vec{r})\right] \nabla \phi=0,
$$

where $s=\varepsilon_{2} /\left(\varepsilon_{2}-\varepsilon_{1}\right)$ is the only relevant material parameter in the problem. Here $\phi$ is the electrical potential and $\eta(\vec{r})$ is the characteristic function for the solid component, defined as having the value 1 at those spatial points occupied by the solid particles, and zero otherwise. The formal solution to Eq. (1), given the condition of $\Delta \phi / \ell=E=1$ in the $z$ direction, can be expressed in the operator notation as

$$
\phi=-\frac{z}{1-\Gamma / s}=-s \frac{z}{s-\Gamma},
$$

where

$$
\Gamma=\frac{1}{V} \int d \vec{r}^{\prime} \eta\left(\vec{r}^{\prime}\right) \nabla^{\prime} G_{0}\left(\vec{r}-\vec{r}^{\prime}\right) \cdot \nabla^{\prime}
$$

is an integral-differential operator, with $G_{0}\left(\vec{r}-\vec{r}^{\prime}\right)=$ $1 / 4 \pi\left|\vec{r}-\vec{r}^{\prime}\right|$ denoting the Green's function for the Laplace equation, and $V$ the sample volume. By defining the inner product operation as

$$
\langle\phi \mid \psi\rangle=\int d \vec{r}^{\prime} \eta\left(\vec{r}^{\prime}\right) \nabla^{\prime} \phi^{*} \cdot \nabla^{\prime} \psi,
$$


it becomes possible to write the effective dielectric constant as

$$
\begin{aligned}
\frac{\bar{\varepsilon}_{z z}}{\varepsilon_{2}} & =-\frac{1}{V} \int d \vec{r}\left(1-\frac{1}{s} \eta\right) \frac{\partial \phi}{\partial z} \\
& =1+\frac{1}{s}\langle z \mid \phi\rangle \frac{1}{V} .
\end{aligned}
$$

From Eqs. (2) and (5), it follows that the effective dielectric constant is given by the Bergman-Milton representation [6]:

$$
\begin{aligned}
\frac{\bar{\varepsilon}_{z z}}{\varepsilon_{2}} & =1-\frac{1}{V} \sum_{n} \frac{\left|\left\langle z \mid \phi_{n}\right\rangle\right|^{2}}{s-s_{n}}=1-\sum_{n} \frac{f_{n}^{z}}{s-s_{n}} \\
& =1-\frac{1}{V} \sum_{n, m}\left\langle z \mid \psi_{n}\right\rangle\left\langle\psi_{n}\left|\frac{1}{s-\Gamma}\right| \psi_{m}\right\rangle\left\langle\psi_{m} \mid z\right\rangle,
\end{aligned}
$$

where $s_{n}$ and $\phi_{n}$ are the $n$th eigenvalue and eigenfunction of the operator $\Gamma$, and $\left\{\psi_{n}\right\}$ is an arbitrary complete basis set. Since the matrix inversion operation in Eq. (6) is noted to be required only for a particular diagonal matrix element, the answer may be obtained efficiently by using the recursive Green's function method [7].

The remarkable feature about the representation, Eq. (6), is that the geometric information is separated from the material information, in contrast to approaches that involve the direct numerical solution of the Laplace equation. This separation means that the microstuctural information are given by the spectral function, i.e., by its value $\left|\left\langle z \mid \phi_{n}\right\rangle\right|^{2} / V=f_{n}^{z}$ and the location of the poles $s_{n}$, both of which are known to be real. Furthermore, $s_{n}$ must lie in the interval $[0,1][6]$. Once the spectral function is obtained, it becomes simple to calculate the effective properties and their associated frequency dependencies, as these factors appear only in $s$. If either $\varepsilon_{1}$ or $\varepsilon_{2}$ is complex and frequency dependent, then the resulting $s$, and consequently $\bar{\varepsilon}_{z z}$ will be complex and frequency dependent. Whereas the imaginary part of $\bar{\varepsilon}_{z z}$ characterizes the overall electrical dissipation of the system, the frequency dependence of the real part of $\bar{\varepsilon}_{z z}$ is what gives rise to the frequency dependence of the yield stress and the shear modulus described below. It should be emphasized that the present formulation is rigorous and includes all the multipole interactions and (self-consistent) local field effects.

Provided the electrostatic ground state has a unique local spatial structure, the target configurations for the maximum $\bar{\varepsilon}_{z z}$ can be limited to periodic structures. We have performed numerical calculations of $\bar{\varepsilon}_{z z}$ to fivedecimal accuracy for six periodic structures - the bodycentered tetragonal (BCT), the face-centered cubic, the hexagonal, the body-centered cubic, the simple cubic, and the diamond. It was found that at any given concentration of the particles, body-centered tetragonal has the largest $\bar{\varepsilon}_{z z}$ and face-centered cubic is a close second, with the rest decreasing in the order given above. The fact that
$\mathrm{BCT}$ is the favored structure is in agreement with prior calculations based on dipole interactions [2] as well as more rigorous approaches $[8,9]$. A direct implication of our theory, that $\bar{\varepsilon}_{z z}$ is maximized under a strong electric field, is the nonlinear electrical response of the overall DER system. This nonlinearity in the electrical response has indeed been widely observed in various ER fluids [10].

A separate calculation has been carried out for cylindrical columns of particles arranged in the BCT structure, with the columns arranged in a two-dimensional triangular superlattice. For column diameters ranging from $2 R$ to $30 R$, it is found that the columnar states are always higher in energy than the pure BCT structure, phase separated from the fluid. However, the electrostatic energy differences are at most $0.1 \%$ of the total electrostatic energy. We note that in most experimental situations particles form columns, and the average column cross section is usually not big (on the order of five particles across). The small energy difference between the column state and the pure BCT state means the overall yield stress and the shear modulus (of the DER model in its ordered state, i.e., the $E>E_{c}$ needed for the liquid-solid transition) may be calculated by using the BCT ground state.

In order to calculate the shear modulus and the yield stress, it is necessary to perturb the system away from its lowest electrostatic free energy state. For the BCT structure, shearing in a direction perpendicular to the $z$ axis means not only a tilt of the $c$ axis away from the electricfield direction by an angle $\theta$, but also a distortion [11] in the lattice constants $c$ and $a$ given by $c / R=2 / \cos \theta, a / R=$ $\left[8-\left(c^{2} / 2 R^{2}\right)\right]^{1 / 2}$, shown schematically in the inset of Fig. 1. As a result, under shear the volume fraction of solid spheres in the BCT structure is also $\theta$ dependent, given by $p_{0}(\theta)=4 \pi \cos ^{2} \theta / 3\left(8 \cos ^{2} \theta-2\right)$. For $\theta$ small, $\bar{\varepsilon}_{z z}(\theta)$

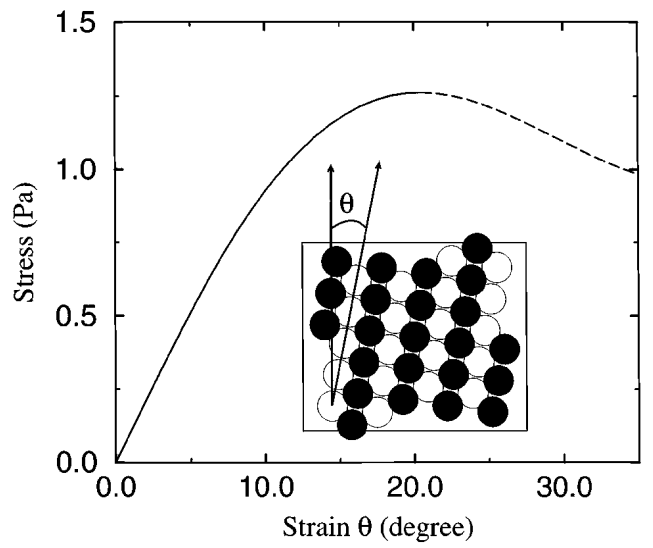

FIG. 1. Calculated stress, in units of Pascal, plotted as a function of strain, i.e., the tilt angle $\theta$ as defined in the inset. The dashed line indicates the unstable regime. The maximum stress is defined as the static yield stress. The parameters used in the calculation are $\varepsilon_{1}=8.4+0.43 i, \varepsilon_{2}=2.71, E=$ $1.32 \mathrm{kV} / \mathrm{mm}$, and $p=0.22$. The inset shows the geometry of the particles under shear. 
may be expanded about its optimal value as

$$
\operatorname{Re}\left[\frac{\bar{\varepsilon}_{z z}(\theta)}{\varepsilon_{2}}\right]=\operatorname{Re}\left[\frac{\bar{\varepsilon}_{z z}(0)}{\varepsilon_{2}}\right]-\frac{1}{2} \mu \theta^{2}+--,
$$

where $\mu$ is the shear modulus in units of $\left|\varepsilon_{2}\right| E^{2} / 8 \pi$. It may be expressed as

$$
\begin{aligned}
\mu=\operatorname{Re} & \left\{\frac{2 p \varepsilon_{2}}{\left|\varepsilon_{2}\right| p_{0}(0)}\left[F_{x}(s)-F_{z}(s)+\frac{\partial F_{z}(s)}{\partial\left(\theta^{2}\right)}\right]_{\mid \theta=0}\right. \\
& \left.+\frac{p}{3 p_{0}(0)}\left[\frac{\bar{\varepsilon}_{\mathrm{BCT}}(0)-\varepsilon_{2}}{\left|\varepsilon_{2}\right|}\right]\right\},
\end{aligned}
$$

with $p$ denoting the volume fraction of solid spheres, $F_{z}(s)=\left\langle z\left|(s-\Gamma)^{-1}\right| z\right\rangle / V, F_{x}(s)=\left\langle x\left|(s-\Gamma)^{-1}\right| x\right\rangle / V$, and $\bar{\varepsilon}_{\mathrm{BCT}}(0)$ being the $\theta=0$ effective dielectric constant of the BCT structure. Because of the vertical nature of the structure, i.e., in either columnar or phase-separation state (column diameter approaching $\infty$ ), $\bar{\varepsilon}_{\mathrm{BCT}}(0)$ is accurately related to the overall effective dielectric constant by the relation

$$
\bar{\varepsilon}_{z z}(0)=\frac{p}{p_{0}(0)} \bar{\varepsilon}_{\mathrm{BCT}}(0)+\left[1-\frac{p}{p_{0}(0)}\right] \varepsilon_{2} .
$$

For the calculation of the yield stress, it is necessary to go beyond the small $\theta$ expansion. Numerical evaluation of the stress-strain relation, i.e., $\operatorname{Re}\left\{\left|\varepsilon_{2}\right|^{-1} \partial \bar{\varepsilon}_{z z}(\theta) / \partial \theta\right\}$ versus $\theta$, is found to display a maximum as shown in Fig. 1. By definition, value of the maximum stress is the static yield stress (in units of $\left|\varepsilon_{2}\right| E^{2} / 8 \pi$ ), as further strain would make the stress decrease, i.e., the structure becomes unstable.

Once the parameters of the spectral function have been calculated, it is simple to evaluate the effective dielectric constant, the shear modulus, the yield stress, and their frequency dependencies. In a first test of the theory, we compare the theoretical prediction in the effective dielectric constant increase with that measured for a simple two-component ER system, consisting of $22 \%$ by volume of monodispersed $1.5 \mu \mathrm{m}$ diameter glass spheres [12] dispersed in silicone oil [13]. The ER fluid sample was baked for $16 \mathrm{~h}$ at $140{ }^{\circ} \mathrm{C}$ in order to remove traces of water, and measured in a narrow-gap cell at $1 \mathrm{kHz}$ and fields up to $1 \mathrm{kV} / \mathrm{mm}$. The saturation value of the high field dielectric constant is found to be 5\% larger than that measured at the low field. By using the measured dielectric constant of glass, $\varepsilon_{1}=6.5$ and silicone oil, $\varepsilon_{2}=2.71$, we get an increase of $5.08 \%$, in excellent agreement with the experiment.

In Fig. 2, we compare the measured frequencydependent static yield stress of the same ER system (solid symbols) with those predicted theoretically (solid lines). The static yield stress was measured by using a standard parallel plates torsional device. With a rms electric field of $1.32 \mathrm{kV} / \mathrm{mm}$ applied across the ER fluid sandwiched between the two parallel plates, the lower plate was rotated slowly, dragging the top plate, until slipping occurred between the two plates. The stress at the point of slipping was taken as the static yield stress. In curve $A$, the high-

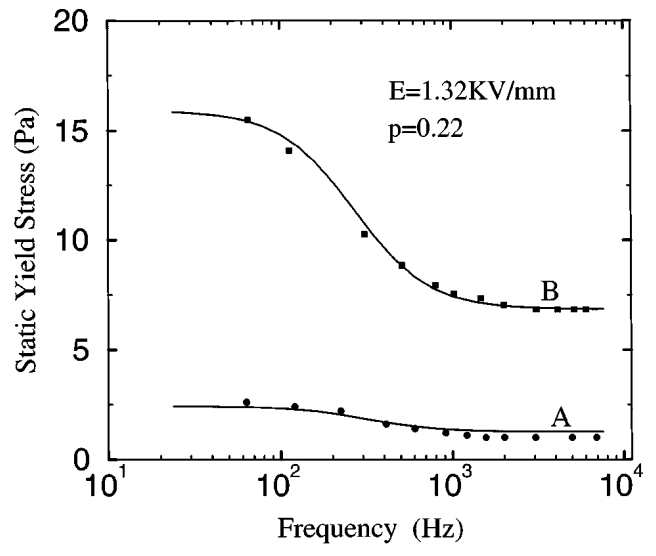

FIG. 2. Comparison between measured (solid symbols) and calculated (solid lines) frequency-dependent static yield stresses, in units of Pascal, for $22 \%$ by volume of $1.5-\mu \mathrm{m}$ glass spheres dispersed in silicone oil. $A$ denotes the system after it has been baked at elevated temperatures to remove trace water. $B$ denotes the same system before water removal. The measurement is done at the rms electric field of $1.32 \mathrm{kV} / \mathrm{mm}$. Parameters used to obtain the calculated results are given in the text.

frequency value of yield stress was calculated with no adjustable parameters. The agreement with experiment is seen to be excellent. To explain the slight frequency dependence, we have used the value of $\tau=4.8 \times 10^{-4} \mathrm{sec}$ and $\varepsilon_{\Delta}=2$ in the Debye form of $\varepsilon_{1}(\omega)$. Curve $B$ gives the yield stress for the same ER system prior to water removal (i.e., baking at elevated temperatures). It is seen that both the value of yield stress and its frequency dependence are much larger. The theoretical curve is calculated by using $\varepsilon_{1}(\omega)=14.8+12 /(1-i \omega \tau)$, with $\tau=6 \times 10^{-4} \mathrm{sec}$ and the measured $\varepsilon_{2}=2.71$. A plausible explanation for the significant effect of trace water is that under a high electric field the water is attracted to the interface between oil and glass, coating the glass particles and thus modifying its effective $\varepsilon_{1}(\omega)$. By using the same parameters and the high-field BCT structure, the calculated conductivity of the system at $10 \mathrm{kHz}$ is $2.5 \times 10^{-8} \mathrm{~S} / \mathrm{m}$. This compares favorably with the measured value of $3 \times 10^{-8} \mathrm{~S} / \mathrm{m}$ at the same frequency. Our results thus demonstrate that the observed frequency dependence of ER fluids' yield stress can be traced to the Debye relaxation processes common in poor insulators, and may be explained quantitatively by the DER model.

The frequency dependence of the shear modulus for the same systems can be directly evaluated from Eq. (8). Here, unfortunately, no experimental data are yet available. The calculated results (solid lines), using the same parameters as those in Fig. 2, show similarity to that of the yield stress. However, when $\varepsilon_{\Delta}$ is large, a novel phenomenon is predicted. That is, over some finite frequency range it is found that the ground state is unstable to a slight elongational distortion of the BCT lattice, and the new ground state is one where the $c$ axis is 


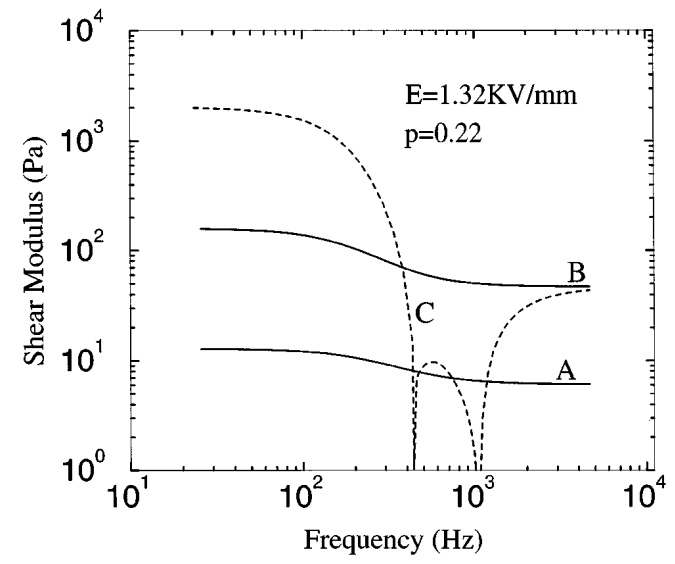

FIG. 3. Calculated frequency dependencies of the shear moduli, in units of Pascal, for the same systems as shown in Fig. 2, shown by the solid lines. The dashed line is calculated by using the same parameters as those of curve $B$, except $\varepsilon_{\Delta}$ is increased to 85 . Soft modes at two frequencies are clearly seen.

elongated and the $a$ axis is correspondingly shortened. The dashed line in Fig. 3 is calculated by using $\varepsilon_{2}=2.71$, $\varepsilon_{1}(\omega)=14.8+85(1-i \omega \tau)$, with $\tau=6 \times 10^{-4} \mathrm{sec}$. It indicates two frequencies where there are soft modes associated with the structural instability $(\mu=0)$. Between these two frequencies the ground state is still a BCT structure, but with the $c$ axis elongated by up to $0.85 \%$, and the $a$ axis shortened. The shear modulus for those frequencies intermediate between the two soft mode frequencies is calculated with respect to the alternate ground state. It is interesting to note that in contrast to the shear modulus, the yield stress calculated with the same parameters gives no hint of any irregularities at finite frequencies. The reason may be gathered from Fig. 1, which indicates that the yield stress is always defined at finite values of $\theta$, and the difference between the two "ground" states is negligible at those tilt angles.

Mathematically, the frequency dependencies of the shear modulus and the yield stress are the direct consequence of the magnitude change in $s$ as the frequency varies. Since the lowest $s_{n}=0$ for touching spheres, the effective dielectric constant, and thus the shear modulus and the yield stress, are enhanced if $|s| \rightarrow 0$ (and vice versa) as seen from Eq. (6). These considerations naturally expose a deep connection between the frequency variation of the rheological properties and their upper bounds, which is a question of considerable practical importance for potential applications of ER fluids. As the value of $|s| \rightarrow 0$, the question of whether the spheres are actually touching becomes crucial. For the purpose of constructing a physical upper bound, we assume that (1) $\left|\varepsilon_{1} / \varepsilon_{2}\right| \rightarrow \infty$, (2) the surfaces of the spheres cannot approach each other closer than a small distance $2 \delta$, e.g., the lower limit on atomic separation (so the lowest $s_{n}$ is slightly greater than 0 ), and (3) $p \leq p_{\text {BCT. With }}$ these conditions, we obtain upper bounds on the yield stress and shear modulus to be $1.38 \sqrt{R / \delta}$ and $1.9(R / \delta)$, respectively, in units of $\left|\varepsilon_{2}\right| E^{2} / 8 \pi$. Taking $R=20 \mu$, $\delta=1 \AA, E=1 \mathrm{kV} / \mathrm{mm}$, and $\varepsilon_{2}=2.5$, we get $8 \mathrm{kPa}$ and $4 \mathrm{MPa}$, respectively, for the two bounds. Since these upper bounds are obtained by setting $s=0$, they are independent of whether we use complex or real $\varepsilon_{1}$ and $\varepsilon_{2}$.

We wish to acknowledge the support of HKUST Research Infrastructure Grant No. RI93/94.SC09 for this work.

*Permanent address: Department of Physics, Jiaotung University, Shanghai, People's Republic of China.

[1] T.C. Halsey and W. Toor, Phys. Rev. Lett. 65, 2820 (1990); T. C. Halsey, Science 258, 713 (1992).

[2] R. Tao and J. M. Sun, Phys. Rev. Lett. 67, 398 (1991); R. Tao and J. M. Sun, Phys. Rev. A 44, R6181 (1991).

[3] T. Garino, D. Adolf, and B. Hance, in Electrorheological Fluids, edited by R. Tao (World Scientific, Singapore, 1992), p. 167; C.A. Randall et al., in Electrorheological Fluids, edited by R. Tao and G. D. Roy (World Scientific, Singapore, 1994), p. 61; R. Liang and Y. Xu, ibid., p. 233; C. A. Randall et al., ibid., p. 516. Deviation from the quadratic field dependence has also been observed and was explained on the basis of the nonlinear fluid conductivity effect. Please see N. Felici et al., ibid., p. 139; L. C. Davis and J. M. Ginder, in Progress in Electrorheology, edited by Kathleen O. Havelka and Frank E. Filisko (Plenum Press, New York, 1995), p. 107.

[4] P. Sheng, Introduction to Wave Scattering, Localization, and Mesoscopic Phenomena (Academic Press, New York, 1995), Chap. 3.

[5] L.D. Landau and E. M. Lifshitz, Electrodynamics of Continuous Media (Pergamon Press, New York, 1984), p. 50 .

[6] D. J. Bergman, in Solid State Physics, edited by H. Ehrenreich and D. Turnbull (Academic Press, New York, 1992), Vol. 46, p. 147; G. W. Milton, Appl. Phys. A 26, 1207 (1981); G. W. Milton, J. Appl. Phys. 52, 5286 (1981).

[7] V. Heine, in Solid State Physics, edited by H. Ehrenreich, F. Seitz, and D. Turnbull (Academic Press, New York, 1980), Vol. 35, p. 1.

[8] G. Bossis, H. Clercx, Y. Grasselli, and E. Lemaice, in Electrorheological Fluids, edited by R. Tao and G. D. Roy (World Scientific, Singapore, 1994), p. 153; H. Clercx and G. Bossis, Phys. Rev. E 48, 2721 (1993).

[9] L. C. Davis, Appl. Phys. Lett. 60, 319 (1992); L. C. Davis, J. Appl. Phys. 72, 1334 (1992); L. C. Davis, J. Appl. Phys. 73, 680 (1993).

[10] K. M. Blackwood et al., in Electrorheological Fluids, edited by R. Tao and G. D. Roy (World Scientific, Singapore, 1994), p. 3; Y.H. Shih and H. Conrad, ibid., p. 294.

[11] Here the distortion is treated as symmetrical in the $x$ and $y$ directions since that is lower in free energy than any distortions that are anisotropic in $x$ and $y$.

[12] Purchased from Nippon Shokubai, product KE-P150.

[13] Purchased from Edwards, product 705. 